\title{
THE NEGATIVE IMPACT OF THE COVID-19 ON ECONOMICS (ON THE EXAMPLE OF GEORGIA)
}

\author{
NIKA ASANIDZE \\ PhD student \\ Ivane Javakhishvili Tbilisi State University, Georgia \\ nikaasanidze@gmail.com
}

\begin{abstract}
Assessing the impact of the COVID-19 crisis on societies, economies and vulnerable groups is fundamental to inform and tailor the responses of governments and partners to recover from the crisis and ensure that no one is left behind in this effort. The world is facing the dangers posed by the global spread of the new COVID-19 which has created new set of problems for the economy. The Crisis has caused many difficulties for Georgia and its economy. The impact of the new virus is rippling through Georgian economy, but it might take a few months to fully see the fallout in data that track economic performance in Georgia. The coronavirus pandemic is putting enormous pressure on healthcare systems, it is affecting the global economy in an unprecedented way and it is leading to a downturn incomparable to any other economic crisis in recent history. It is hard to estimate the depth and duration of the recession for every region and country. It will depend not only on the development of pandemic, but also on specific economic futures, strengths and vulnerabilities. Today the economy is a hostage to medicine. In Georgia, had on the following industries: tourism, transportation, agriculture and real estate had the biggest negative impact from COVID-19. Hotels, restaurants and tourist agencies demand from the government to postpone the budget taxes and bank loans. Meanwhile the hospitality sector of Georgia tries to minimize the cost because of sharp drop in revenue. Different countries will need different responses to dealing with the global economic challenges. As a fact COVID-19 has taught many countries to survive during the crisis period. From the economic stand point of view, every country has suffered the negative consequences of the COVID-19. The level of the consequences had direct correlation on the economic factors.
\end{abstract}

KEYWORDS: PANDEMIC, ECONOMIC GROWTH, SECTORAL ANALYSIS, GLOBAL ECONOMIC CHALLENGES.

For citation: Asanidze, N., (2020). The Negative Impact of the ovid-19 on Economics (On the Example of Georgia). Globalization and Business, 10. 210-217. https://doi.org/10.35945/gb.2020.10.027 


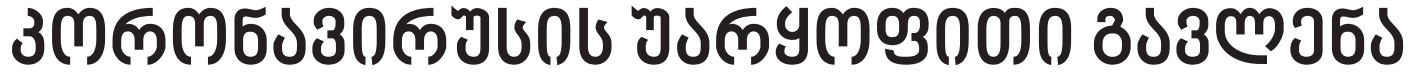

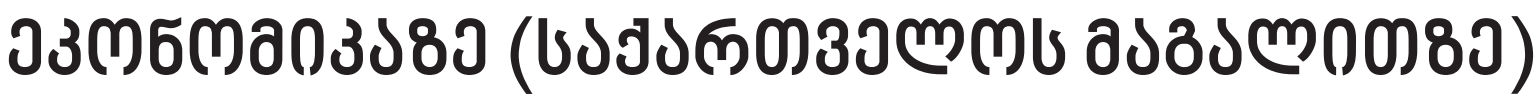

\author{
б03১ ১U১60d0

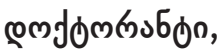

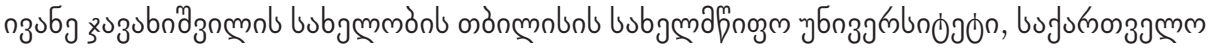 \\ nikaasanidze@gmail.com
}

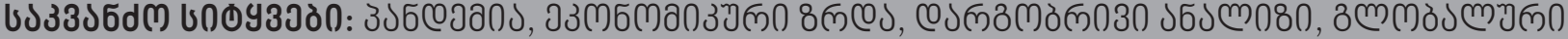

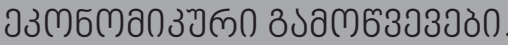

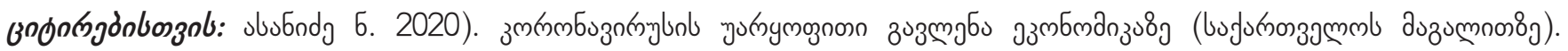

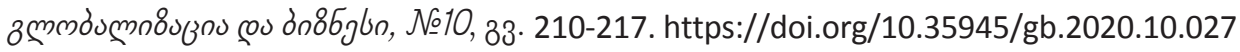

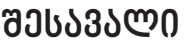

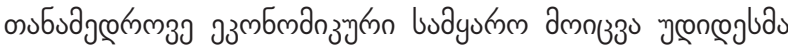

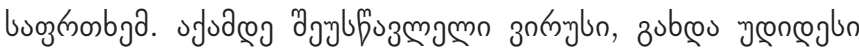

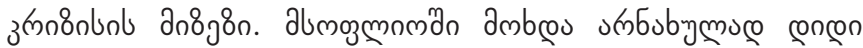
зum

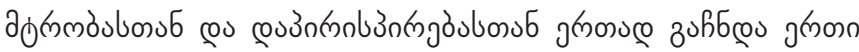
zudugrnon

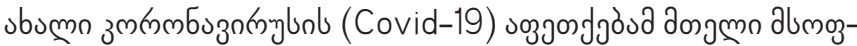

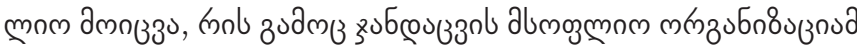

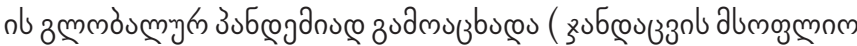

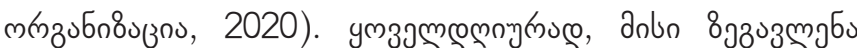

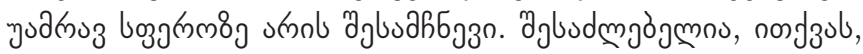

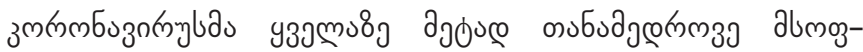

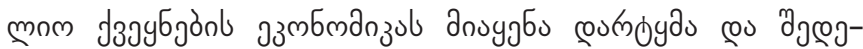
зиме z

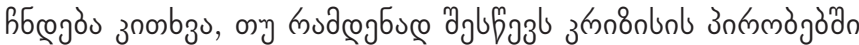

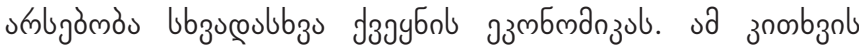

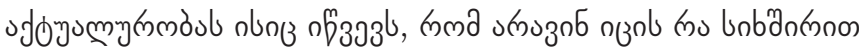

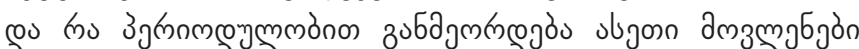

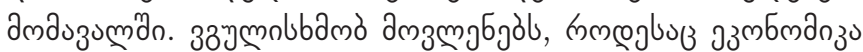

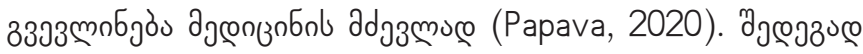

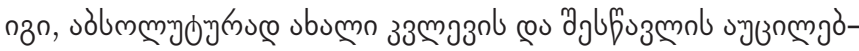

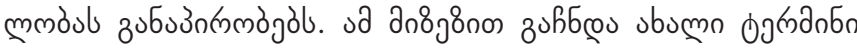

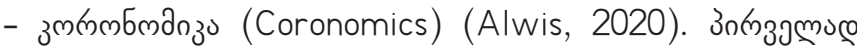

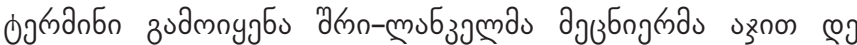

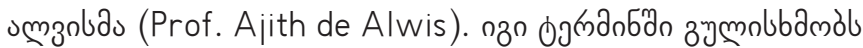

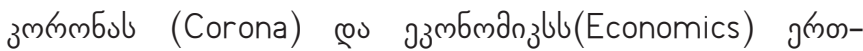

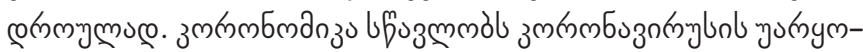

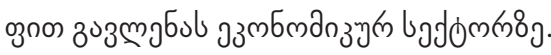

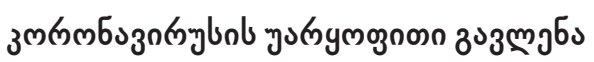

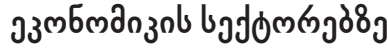

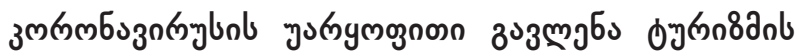

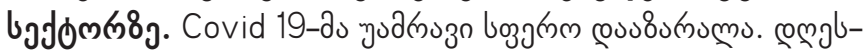

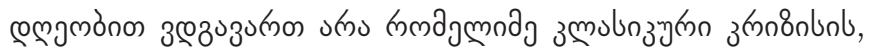

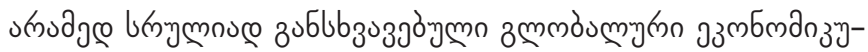

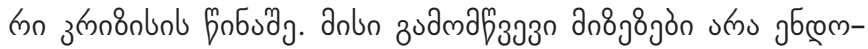

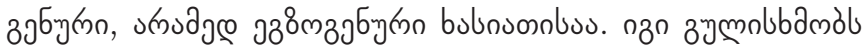

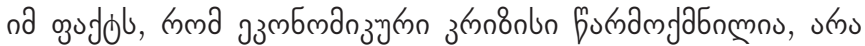

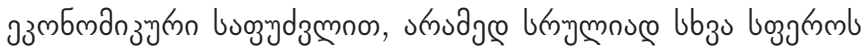

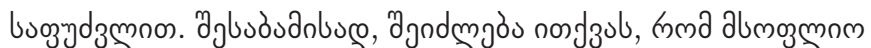

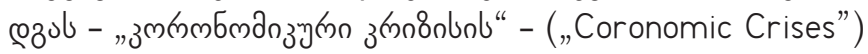
bugnmosbol fabudg (Papava \& Charaia, 2020:4). umbioson

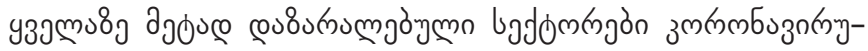

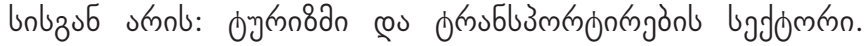
oy

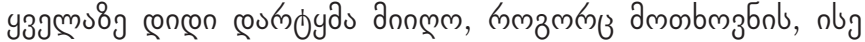

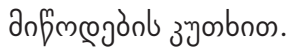

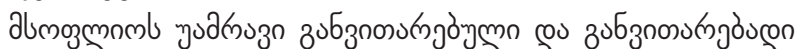

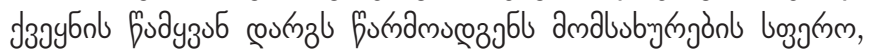

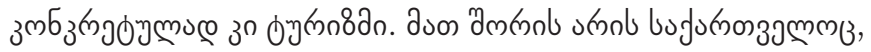

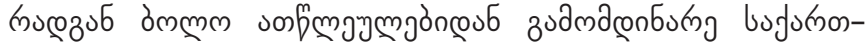

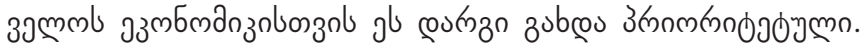

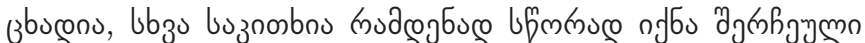

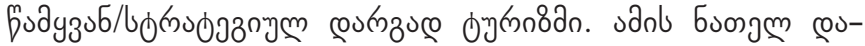

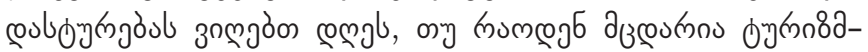

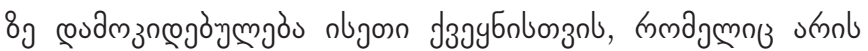

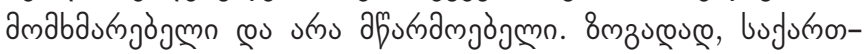

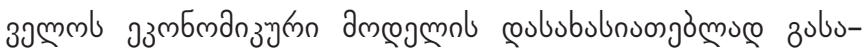




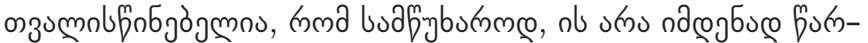

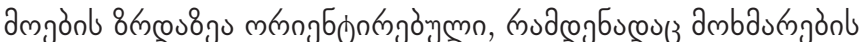
bonaymnn

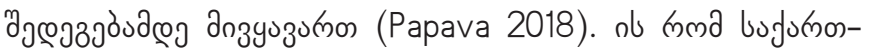

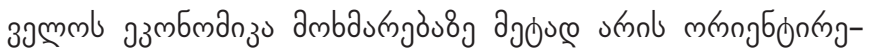

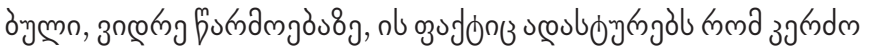

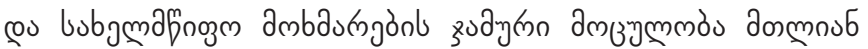

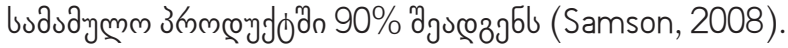

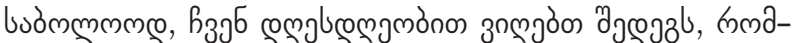

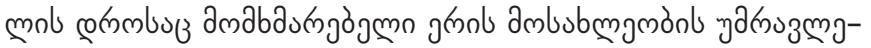

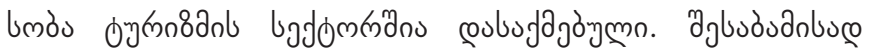

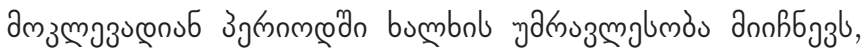

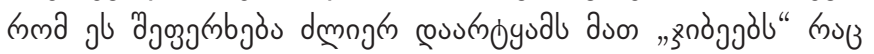

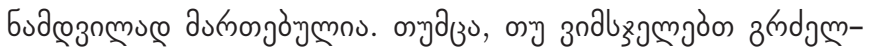

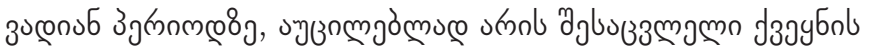

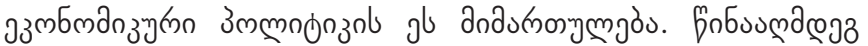

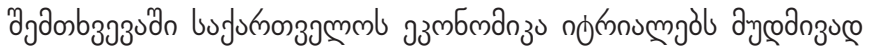

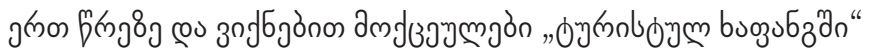

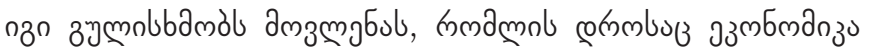

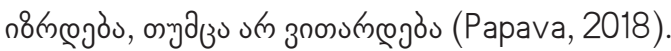

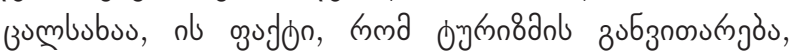

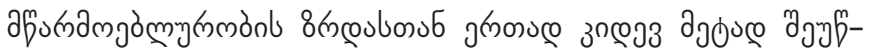

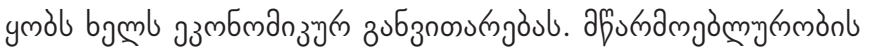

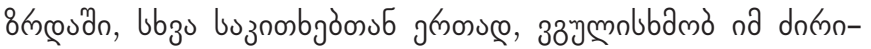

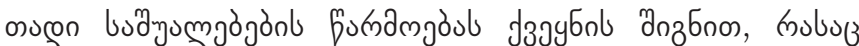

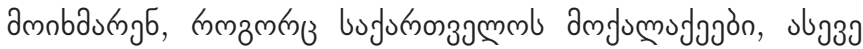

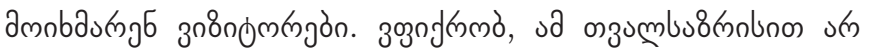

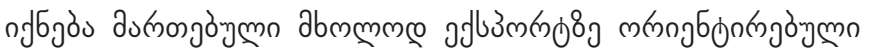

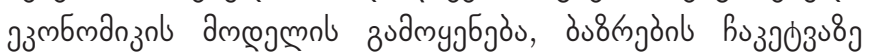

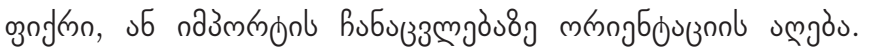

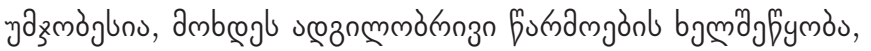

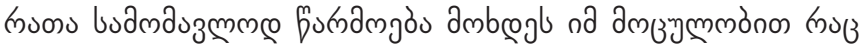

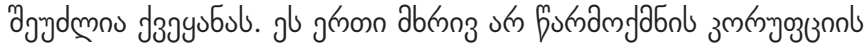

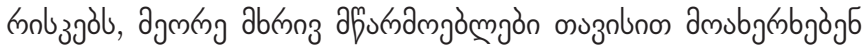

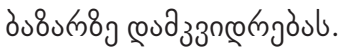

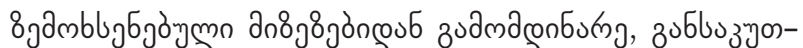

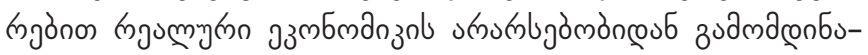

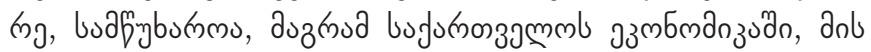

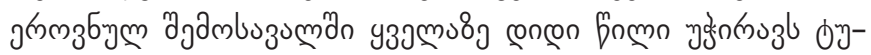

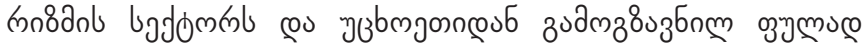

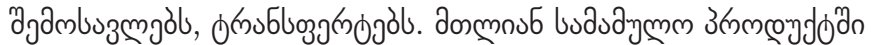

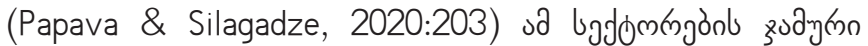

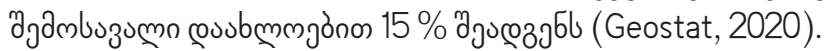

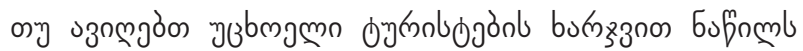

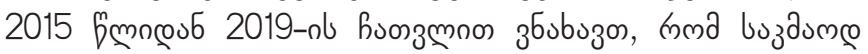

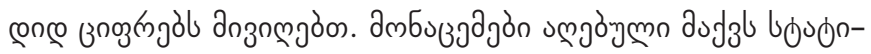

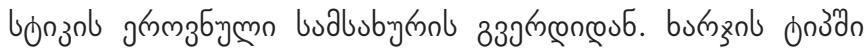

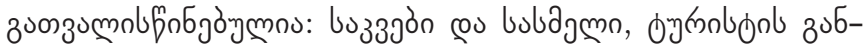

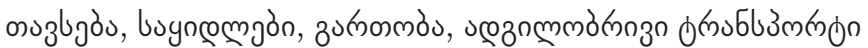
cou bbzu bumgrgòn (Geostat, 2020).

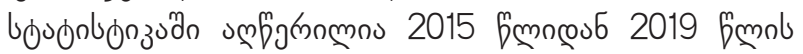

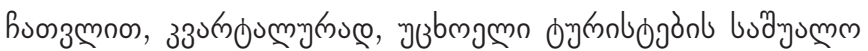

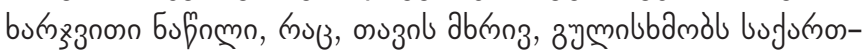

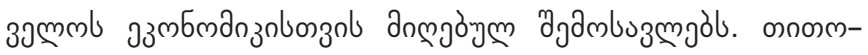

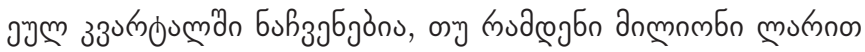

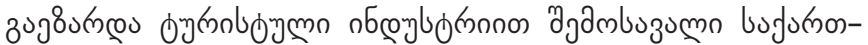
зjmml azmbmanzul. ob guzumnonse:

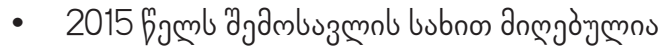

4058400000 mukn;

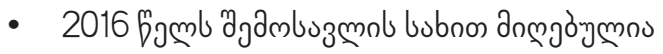

4380000000 mutro;

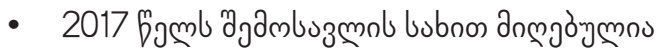

5761200000 mutro;

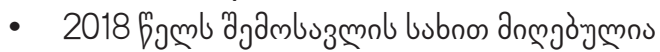

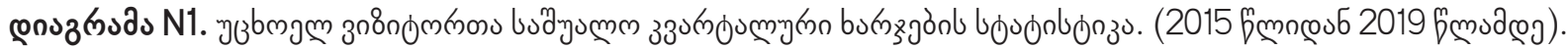

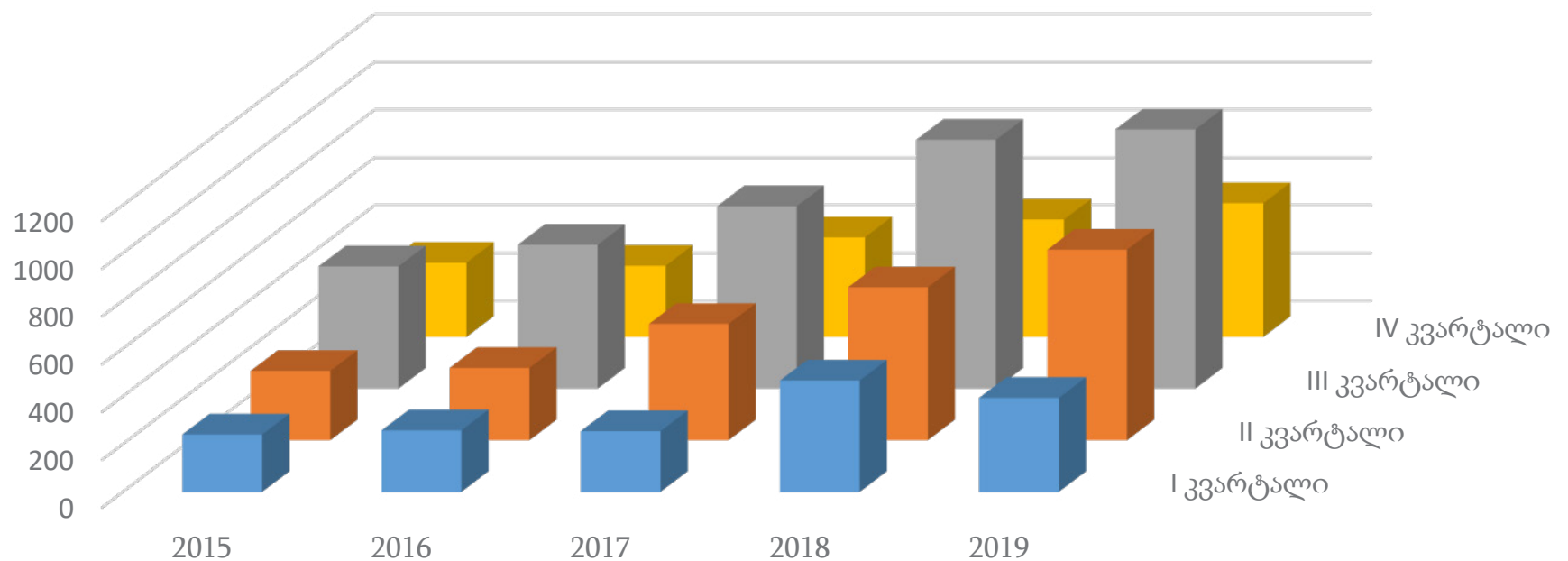

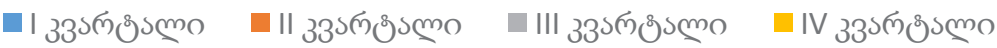




\section{0 munn;}

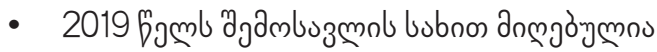
8511600000 muKno.

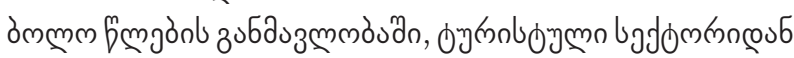

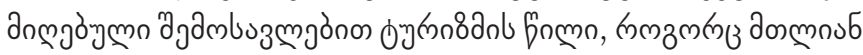

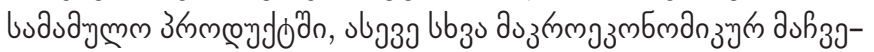

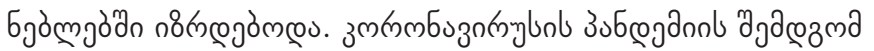

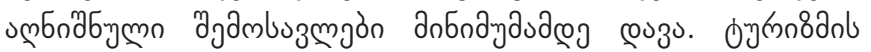

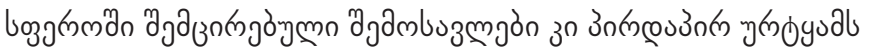

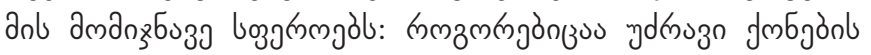

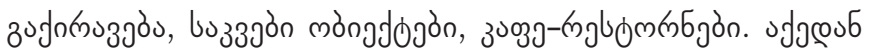

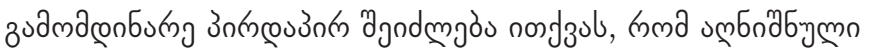

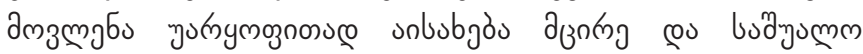

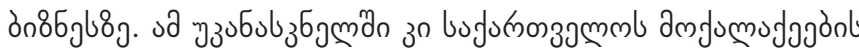
dumnob conen Eufिnmo gुgenl.

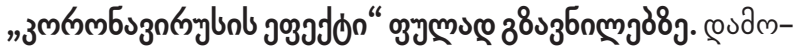

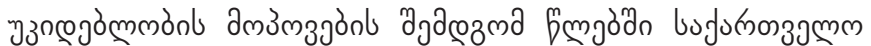

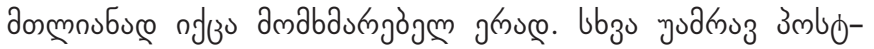

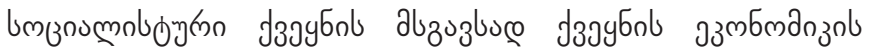

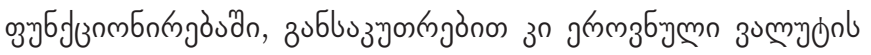

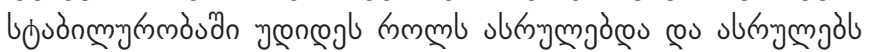

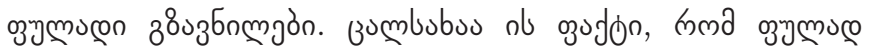

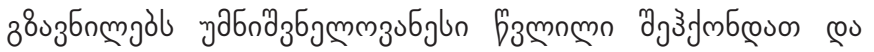

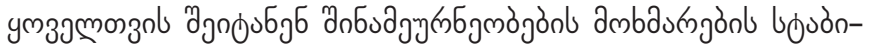

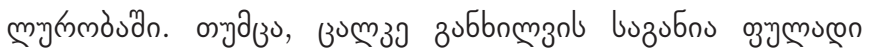

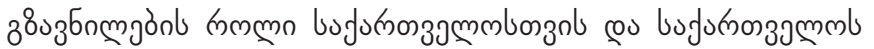

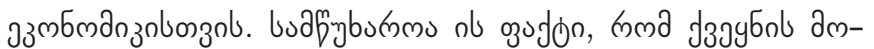

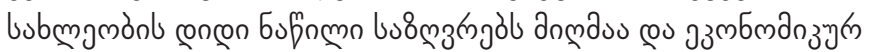

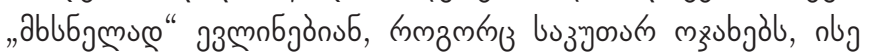

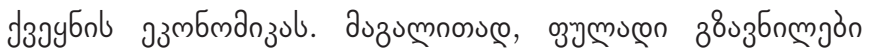

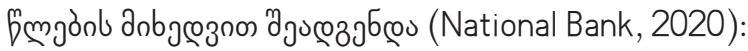

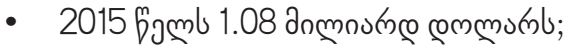

- 2016 fambl 1.1 anmouńn commúnb;

- 2017 Gambl 1.4 anmnukne commúrb;

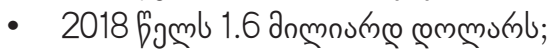

- 2019 fambl 1.7 anmonume commúnt.

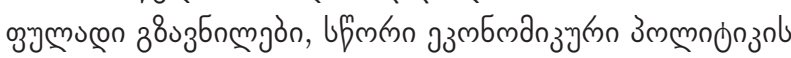

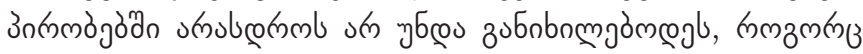

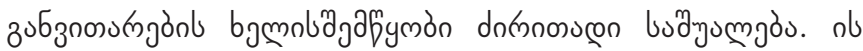

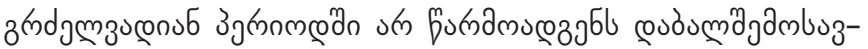

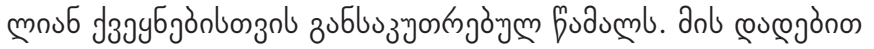

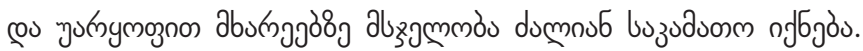

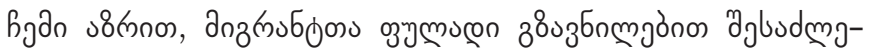

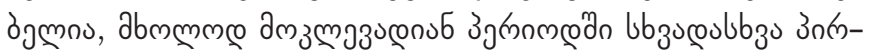

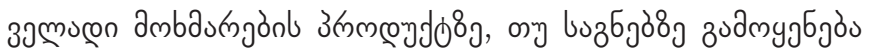

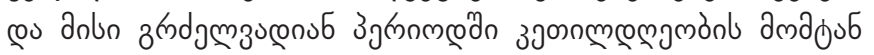

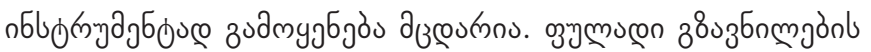

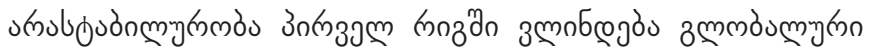

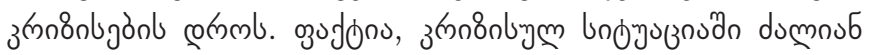

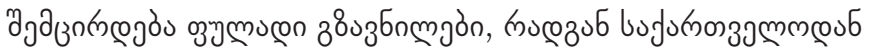

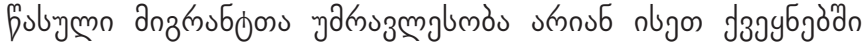

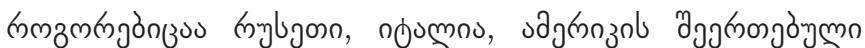

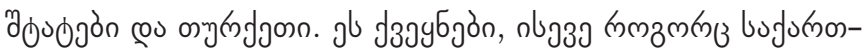

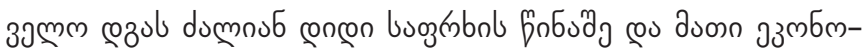

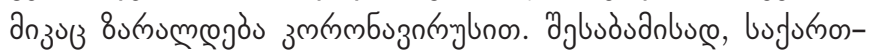

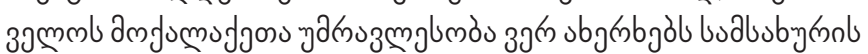

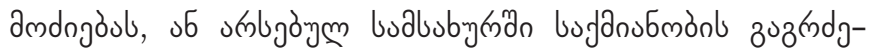

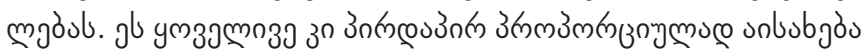

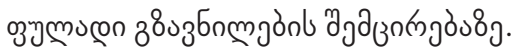

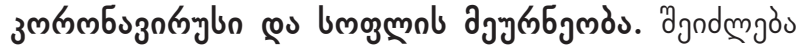

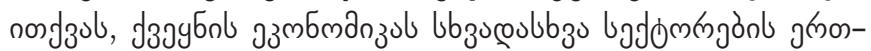

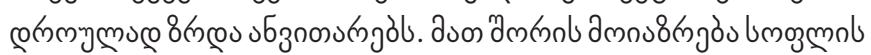

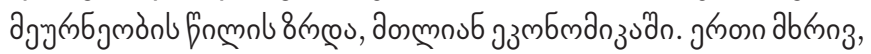

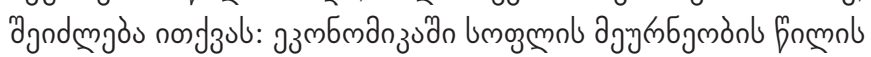

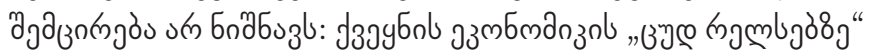

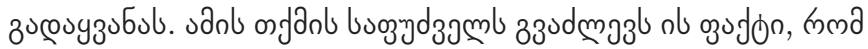

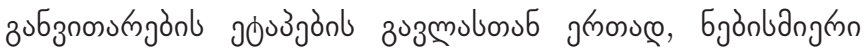

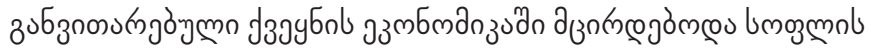

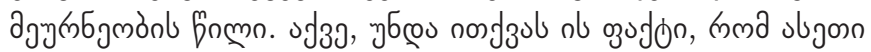

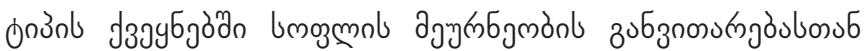

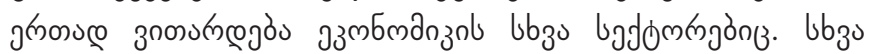

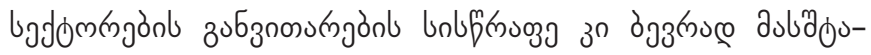

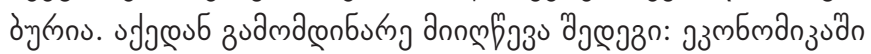

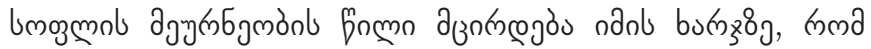

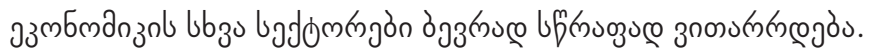

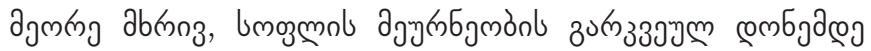

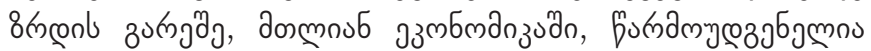

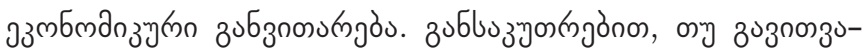

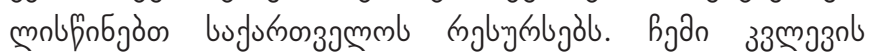

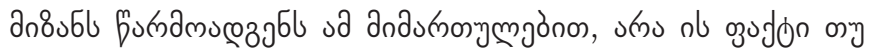

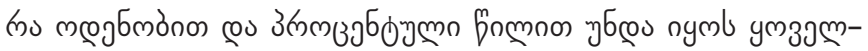

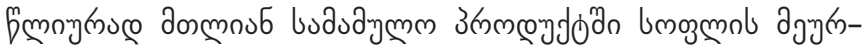

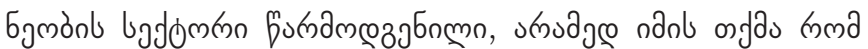

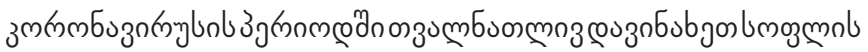

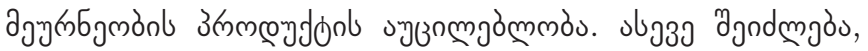

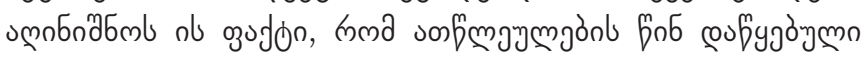

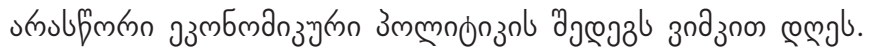

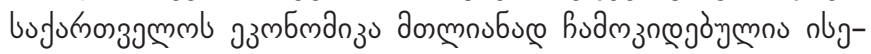

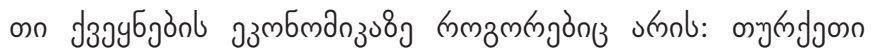

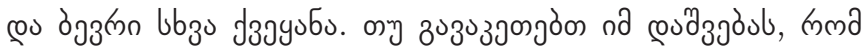

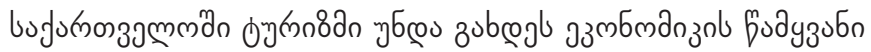

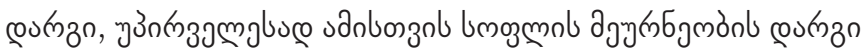

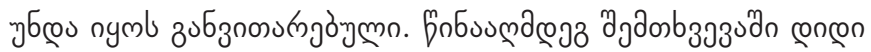

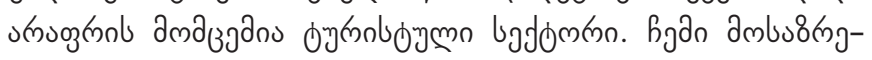

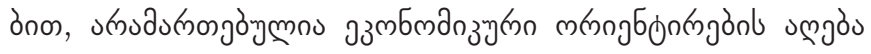

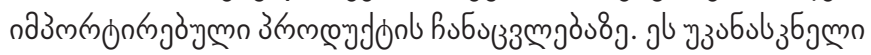

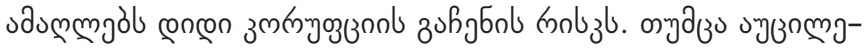

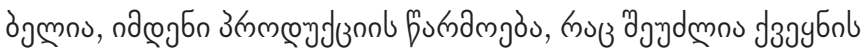
эзмбmanzul.

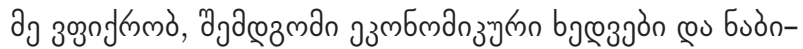




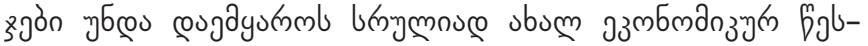

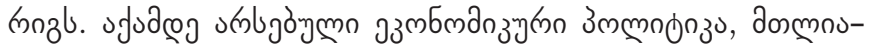

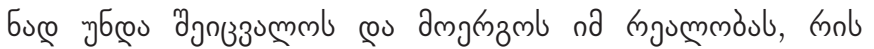

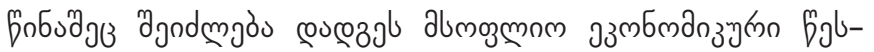

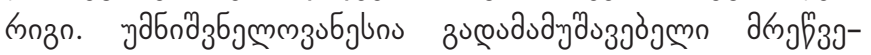

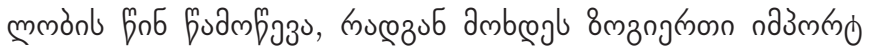

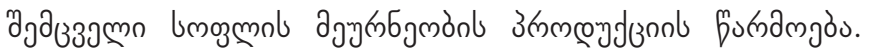

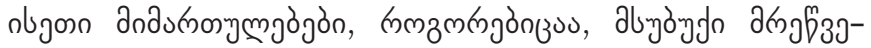

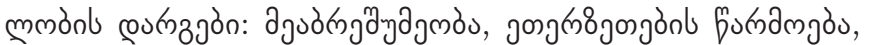

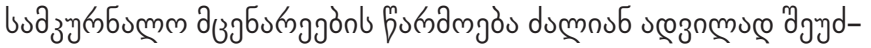

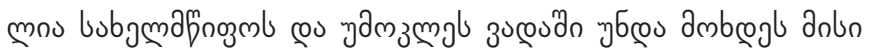

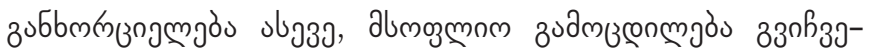

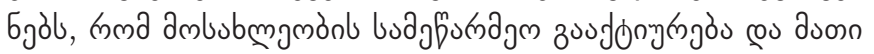

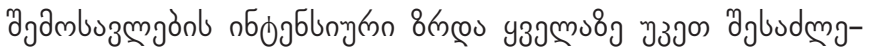

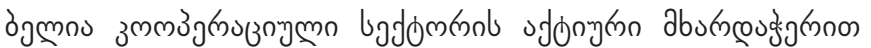
(Koguashvili, Papava, Silagadze, Kunchulia, Mekvabishvili, Gogokhia, Chikhladze, Ramishvili, Archvadze, Galegashvili, 2020).

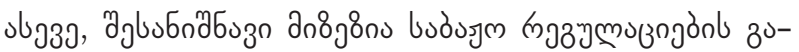

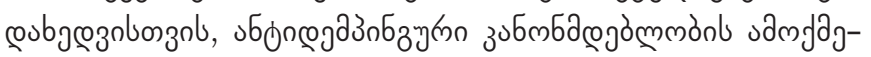

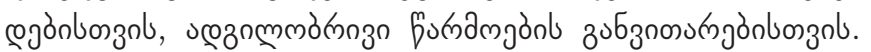

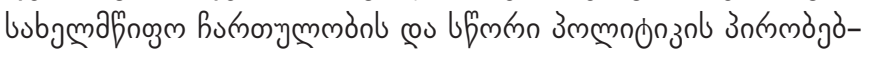

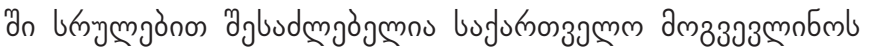

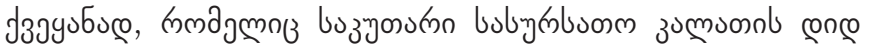

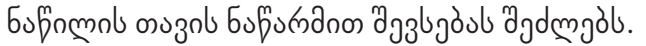

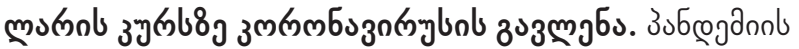

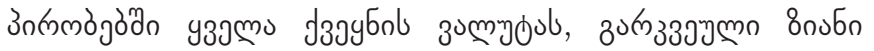

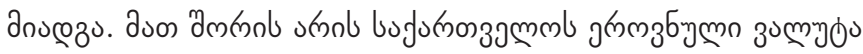

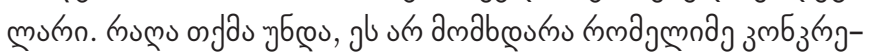

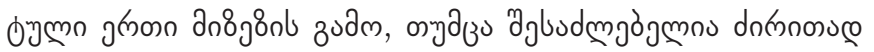

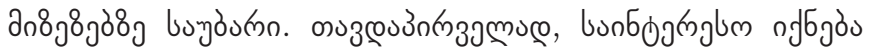

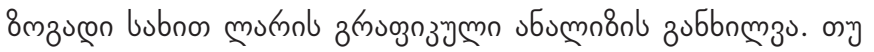

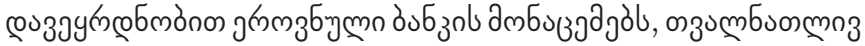

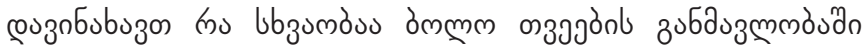

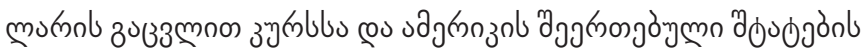

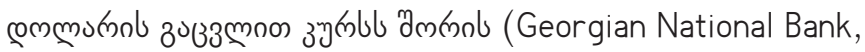

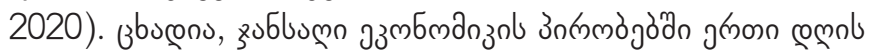

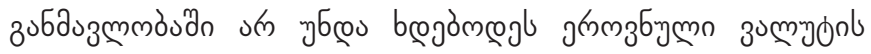

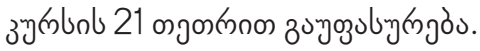

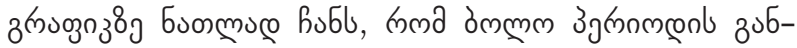

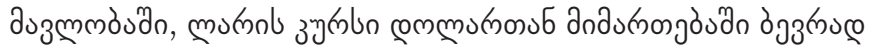

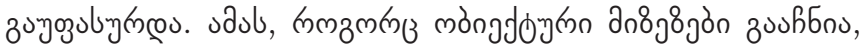

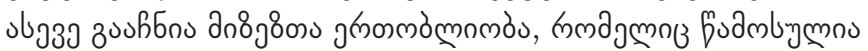

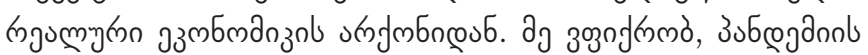

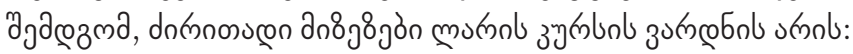

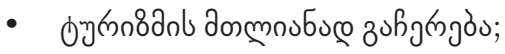

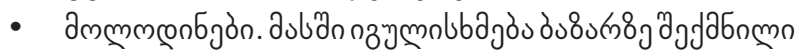

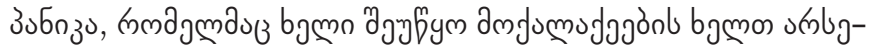

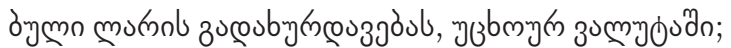

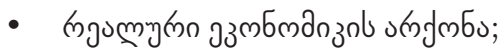

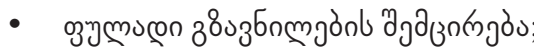

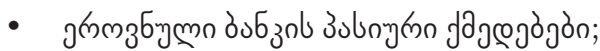

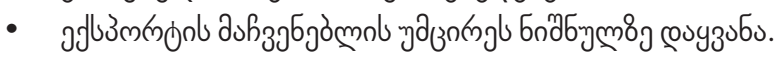

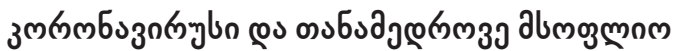

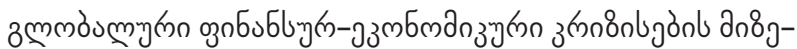

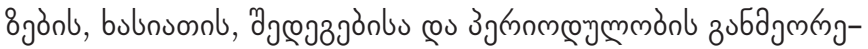

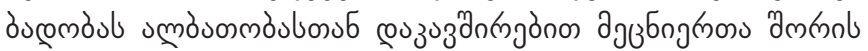

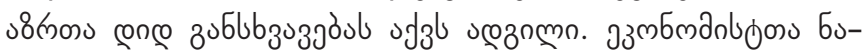

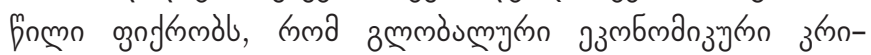

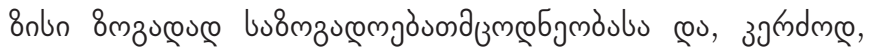

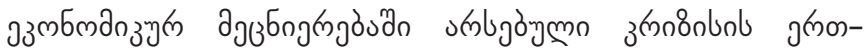

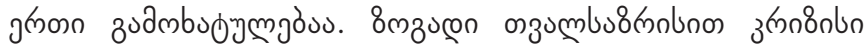

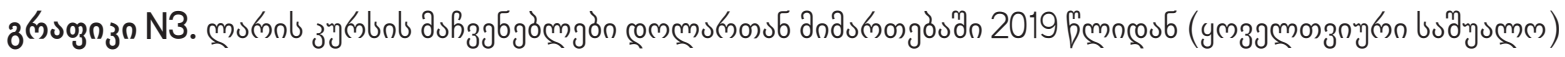

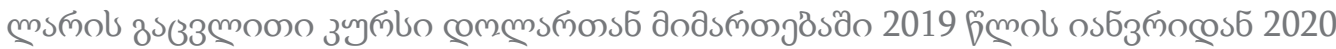

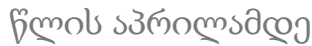

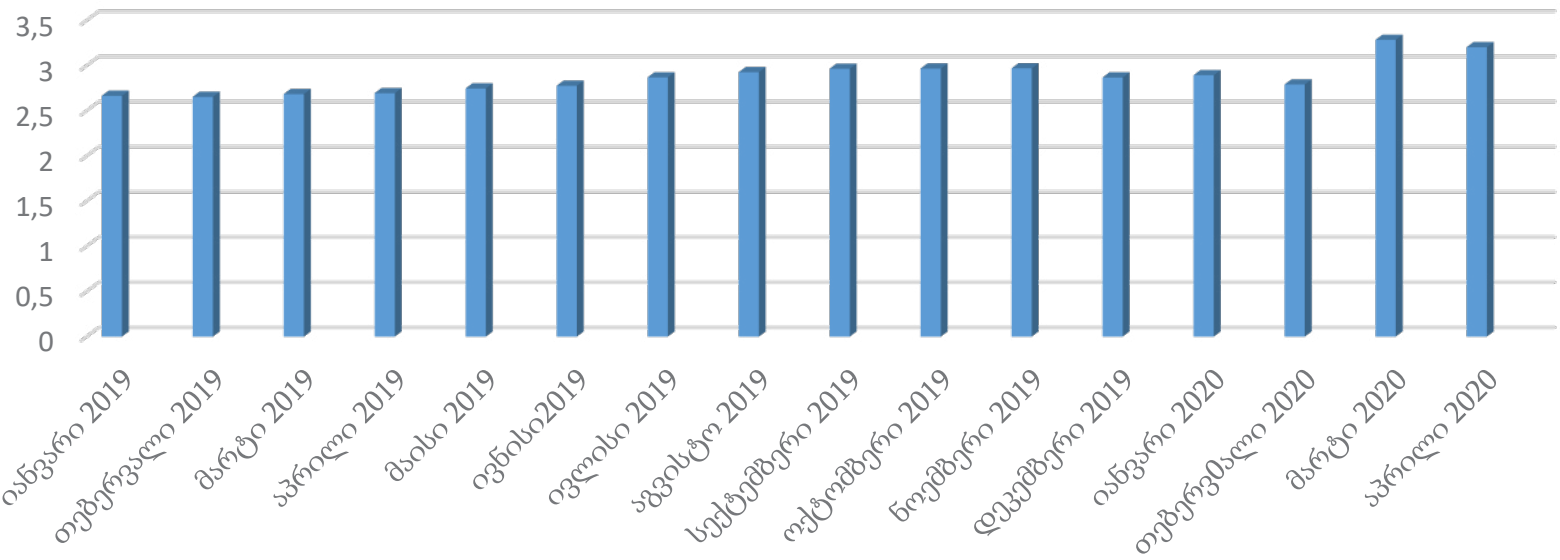




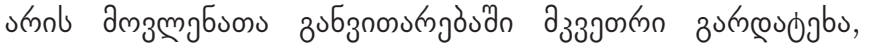

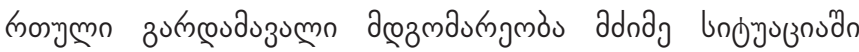

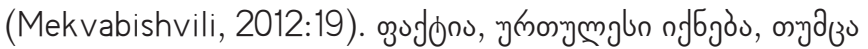

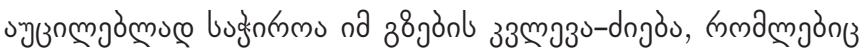

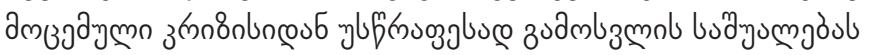

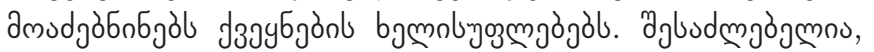

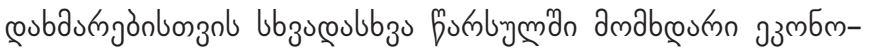

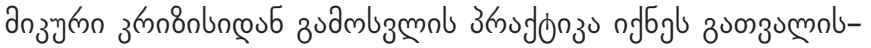

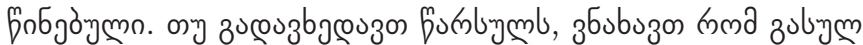

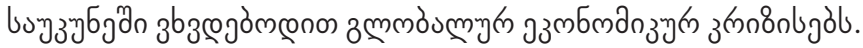
àzumnooso:

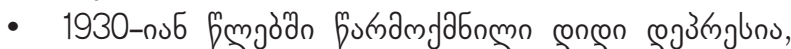

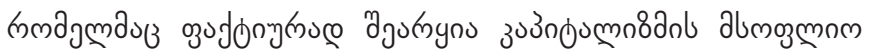

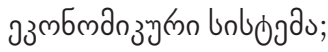

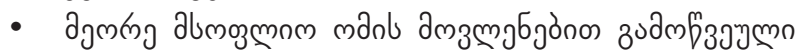

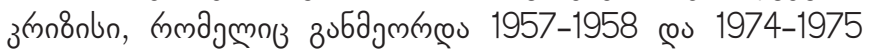

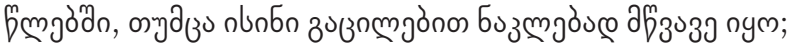

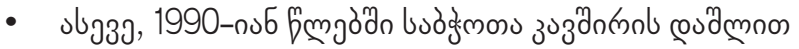

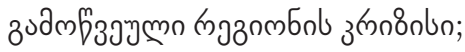

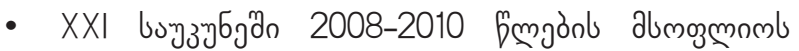

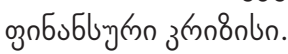

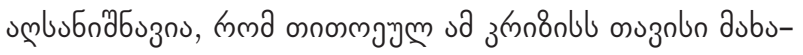

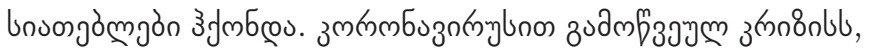

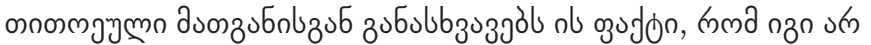

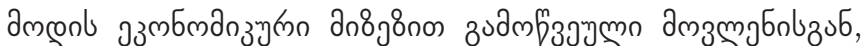

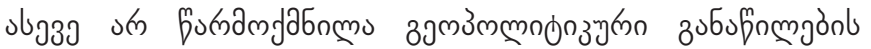

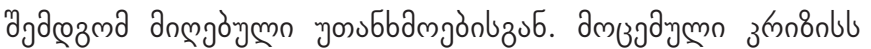

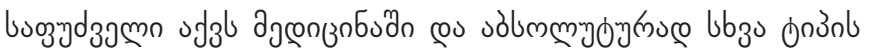

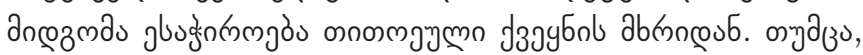

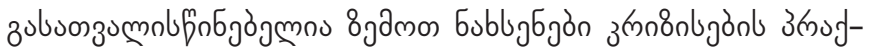

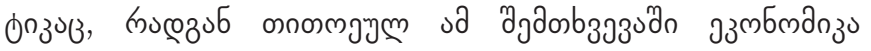

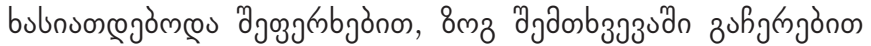

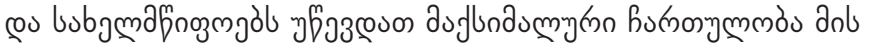

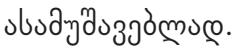

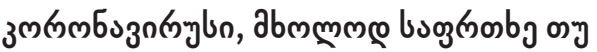

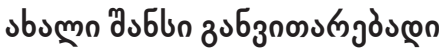

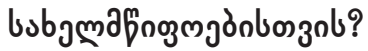

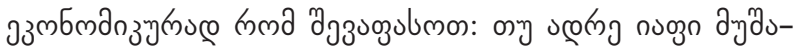

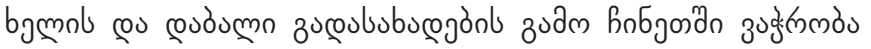

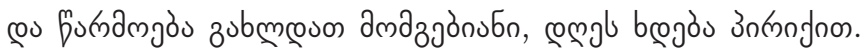

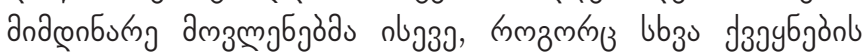

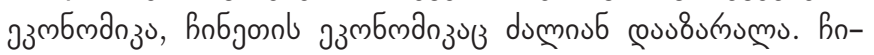

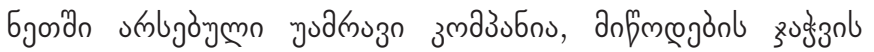

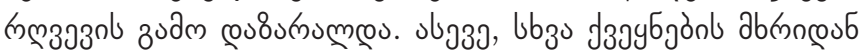

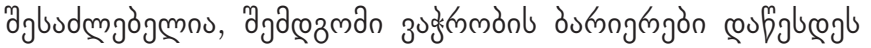

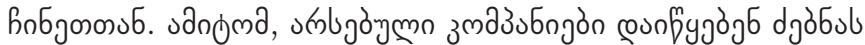

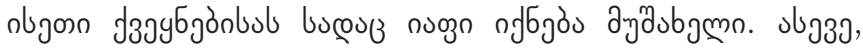

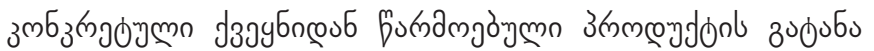

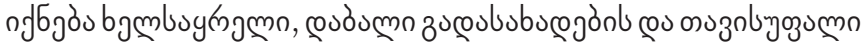

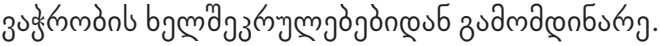

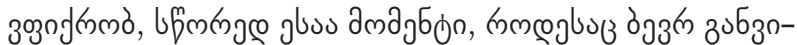

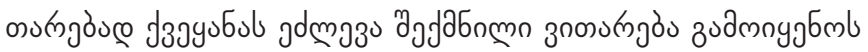
zuбz

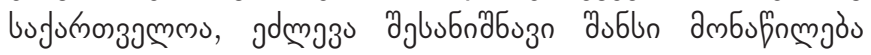

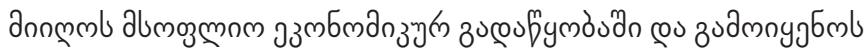

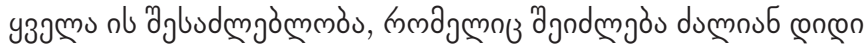

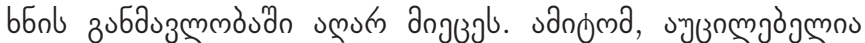

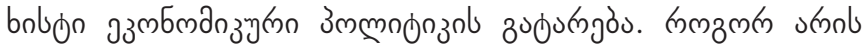

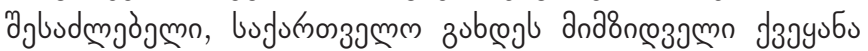

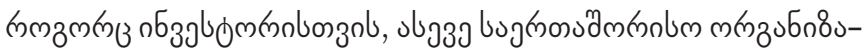

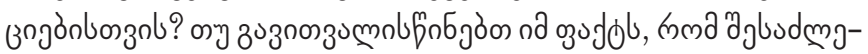

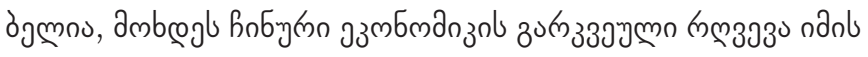

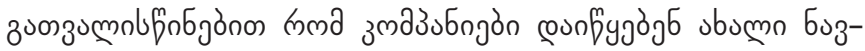

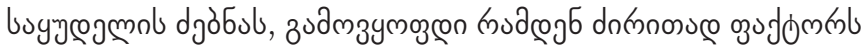

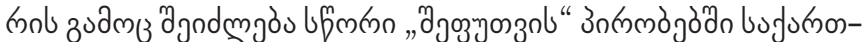

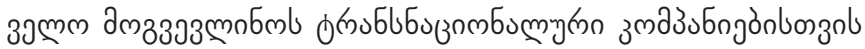
bunбoj

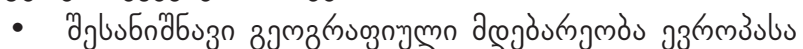
cou vonul gmmols;

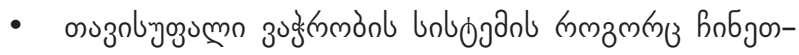

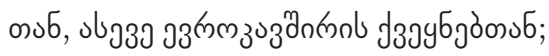

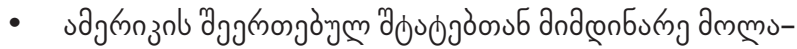

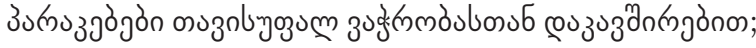

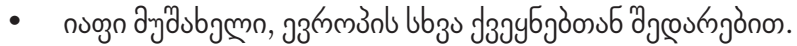

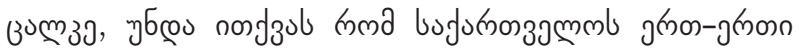

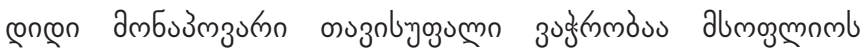

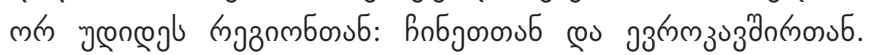

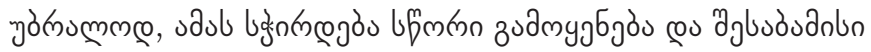

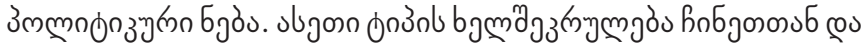

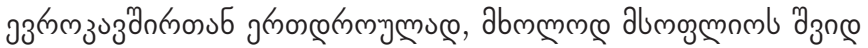

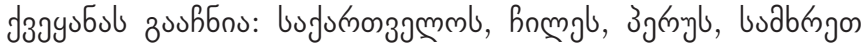

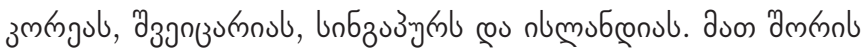

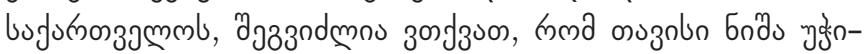

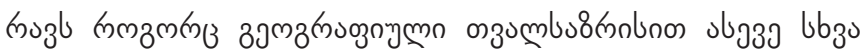

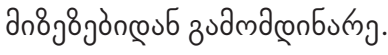

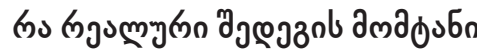

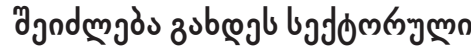

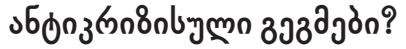

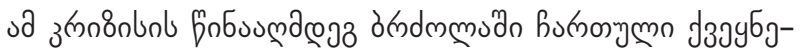
on bygmadmзuб

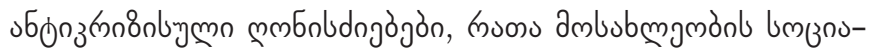

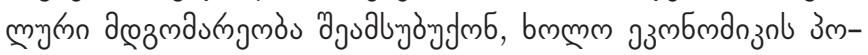

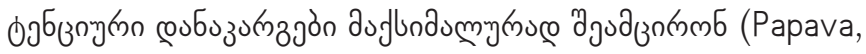
2020).

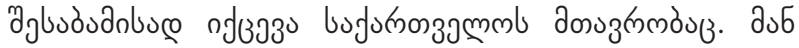

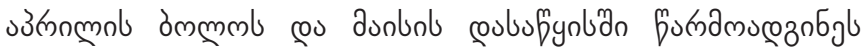




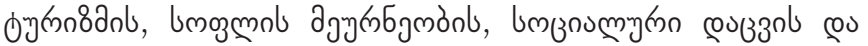

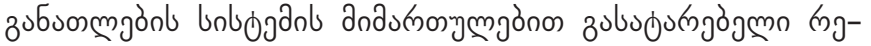

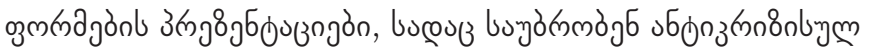

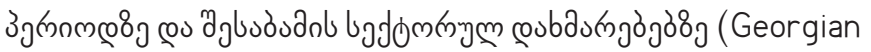

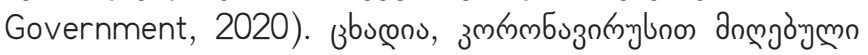

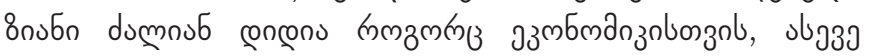

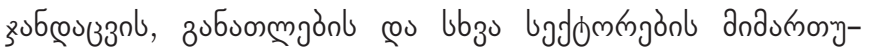

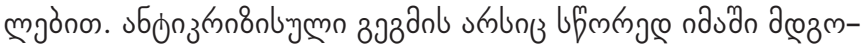

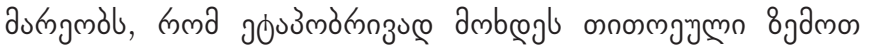

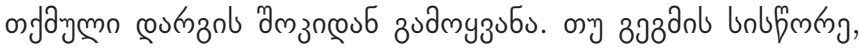

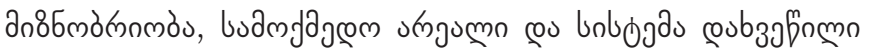

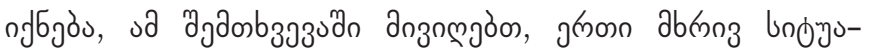

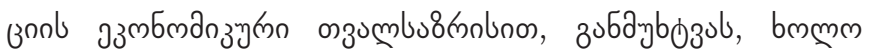

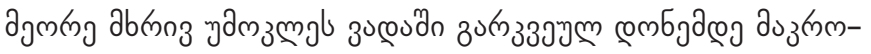

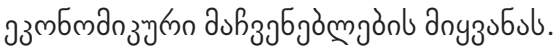

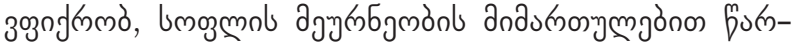

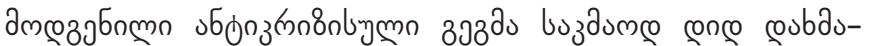

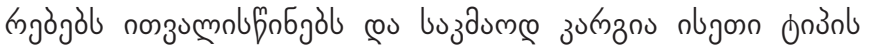

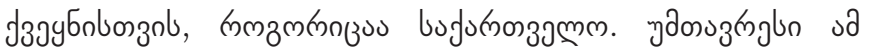

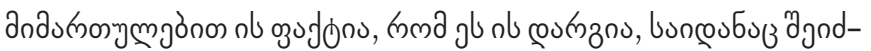

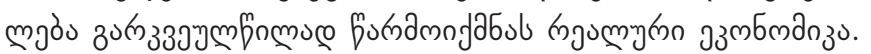

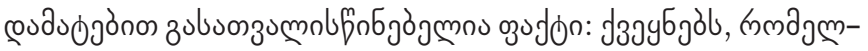

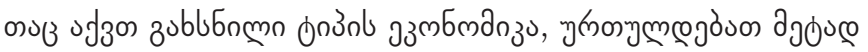

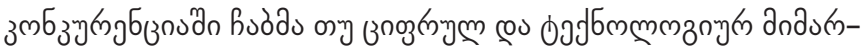

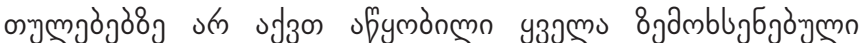

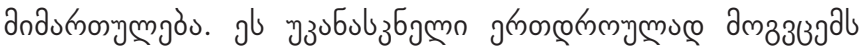

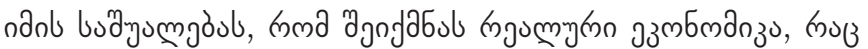

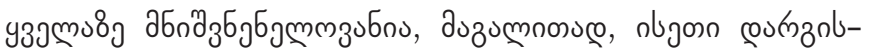

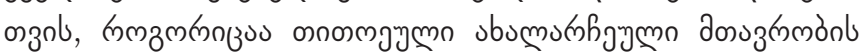

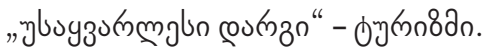

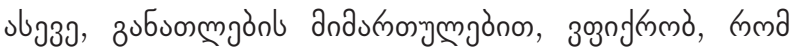

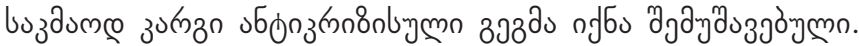

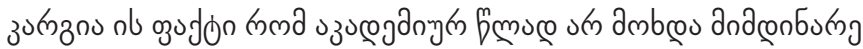

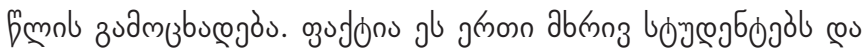

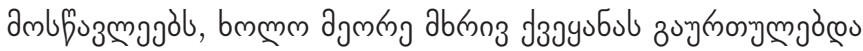

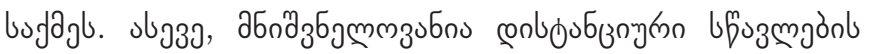

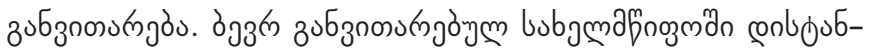

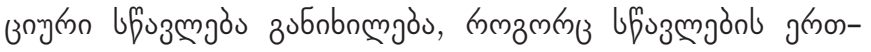

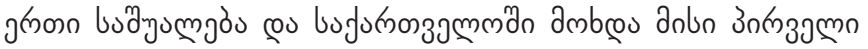

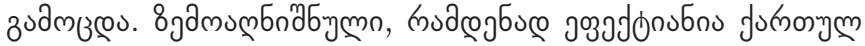

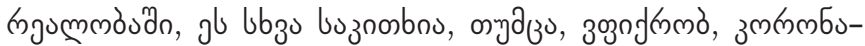

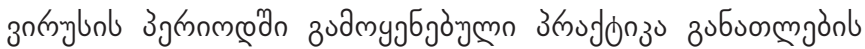

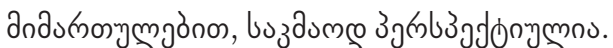

\section{esu336s}

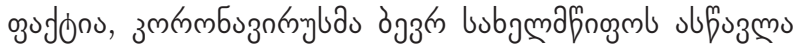

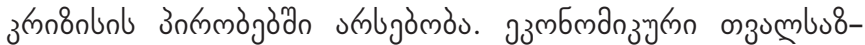

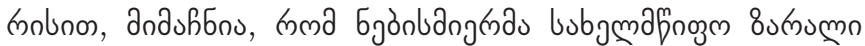

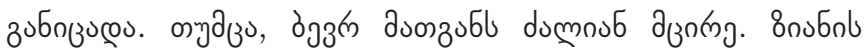

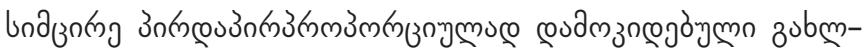

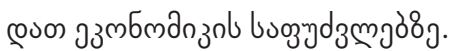

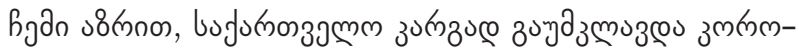

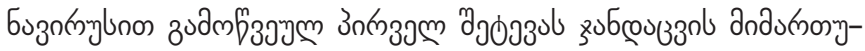

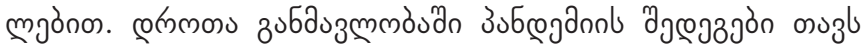

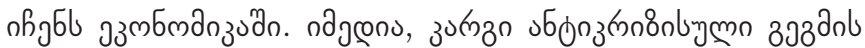

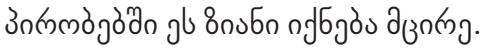

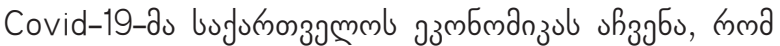

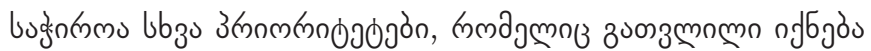

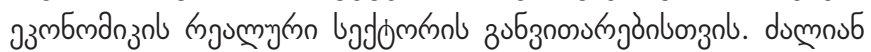

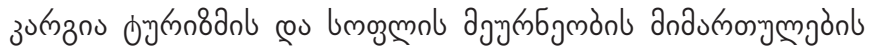

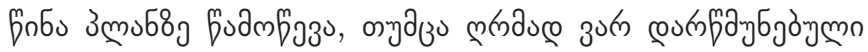

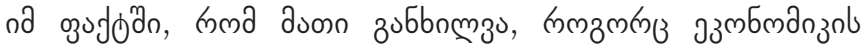

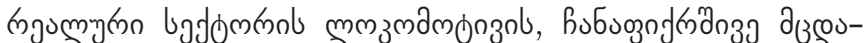

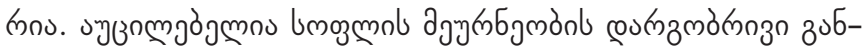

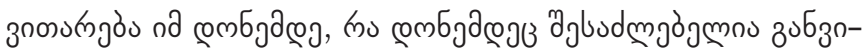

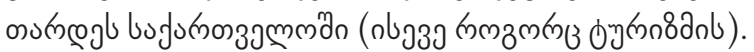

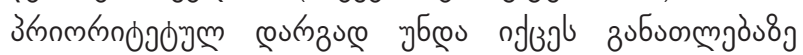

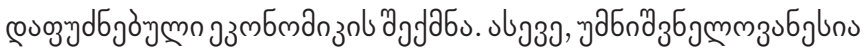

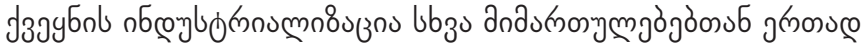

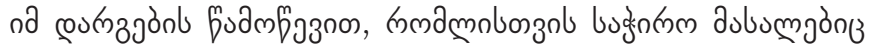
ibzugou bufunorzamman.

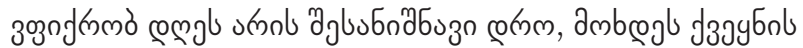

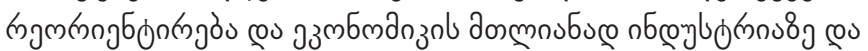

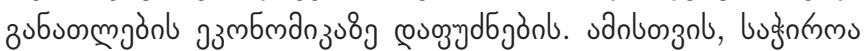

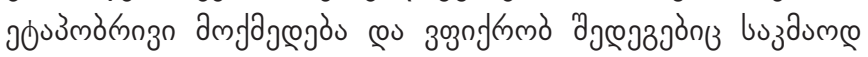
zurnan nfбjós.

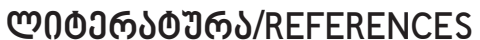

Koguashvili, P., Papava, V., Silagadze, A., Kunchulia, T., Mekvabishvili, E., Gogokhia, R., Chikhladze, N., Ramishvili, B., Archvadze, I. \& Galegashvili, D. (2020). We will defeat Korona. Hunger and Poverty? Resonance. (In Georgian); http:// www.resonancedaily.com/mobile/index.php?id_rub=11\&id_artc=92467

Mekvabishvili, E. (2012). Theory of economic crises and financial-economic crises of the globalization era. Globalization, Modern Problems of International Business and Development Perspectives. Tbilisi, Universal Publishing House; (In Georgian); <https://taxuna.files.wordpress.com/2015/11/unnamed-file.pdf >. 
Papava, V. \& Charaia, V. (2020). The Coronomic Crisis and Some Challenges of the Georgian Economy. Tbilisi: Georgian Strategy and International Relations Research Foundation. Expert Opinion, N136 (In Georgian); <http://papava.info/ publications/Papava_Charaia_coronomical-crisis-and-Georgia.pdf>.

Papava, V. (2018). Georgiass economic model: Consumer paradox and touristic trap. In: The Challenges of Globalization in Economics and Business. Tbilisi, Ivane Javakhishvili Tbilisi State University Publishing House, (In Georgian);

$<$ https://www.tsu.ge/data/file_db/economist_faculty/konference.pdf $>$.

Papava, V., Silagadze A. (2019). How to translate the term «Gross Domestic, Product» into Georgian. Globalization and Business. No 7; (In Georgian); <https://www.researchgate.net/publication/334897039_rogor_unda_itargmnos_ kartulad_termini_GROSS_DOMESTIC_PRODUCT>.

Papava, V. (2020). Pension, Economic Growth, Agflation and Inflation, Georgian Strategy and International Relations Research Center; (In Georgian); < https://www.gfsis.org/ge/blog/view/1071>.

National Bank, (2020). Money transfers in Georgia. National Bank of Georgia; (In Georgian); <https://www.nbg.gov.ge/index. php?m=304>.

National Bank, (2020). Official exchange rates. National Bank of Georgia, (In Georgian); <https://www.nbg.gov.ge/index. php?m=306>.

Geostat, (2020). Distribution of tourist expenses. National Statistics Office; (In Georgian); < https://www.geostat.ge/ka/ modules/categories/101/shida-turizmi>.

Geostat, (2020). The share of agriculture in the total domestic product. National Statistics Office; (In Georgian);

$<$ https://www.geostat.ge/ka/modules/categories/196/soflis-meurneoba>.

Government of Georgia, (2020). Anti-crisis plans for education, tourism and agriculture; (In Georgian); <http://gov.ge/index. php?lang_id=GEO\&sec_id=288>.

Alwis, A. de. (2020). "Coronomics -Plan Your Eggs and the Basket!" Daily FT, February 25, <http://www.ft.lk/columns/ Coronomics-\%E2\%80\%93-Plan-your-eggs-and-the-basket-/4-695109>.

Papava, V. (2020). "Coronomic Crisis: When the Economy Is A Hostage to Medicine." Eurasia Review. Journal of analysis and news. March 29, <https://www.eurasiareview.com/29032020-coronomic-crisis-when-the-economy-is-a-hostage-tomedicine-oped/>.

Papava, V. (2018). Georgia's Economy in a Tourist Trap. Rondeli Blog, July 25 <https://www.gfsis.org/blog/view/854>.

Samson, I. (2008). Medium-Term Prospects for the Georgian Economy. Georgian Economic Trends, February.

WHO Director-General's Opening Remarks at the Media Briefing on COVID-19 - 11 March . (2020). World Health Organization. $<$ https://www.who.int/dg/speeches/detail/who-director-general-s-opening-remarks-at-the-media-briefing-on-covid19---11-march-2020>. 